Electronic supporting information for:

\title{
The Complex Micellization Behavior of the Polysorbates Tween 20 and Tween 80
}

Hannah Knoch, Maximilian H. Ulbrich, Judith J. Mittag, Julia Buske, Patrick Garidel*, Heiko Heerklotz*

1) Comparing demicellization curves in buffer and water, buffer-into-buffer blank

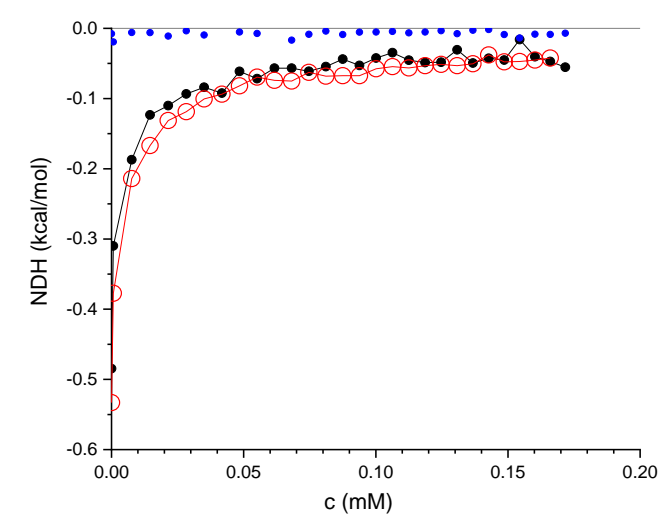

Figure S1. Titrations of $1 \mathrm{mM}$ PS80 in buffer into buffer (black solid spheres), $1 \mathrm{mM}$ PS80 in water into water (red hollow spheres) and of buffer into buffer (blue solid spheres). The injection volumes match the standard protocol used in the manuscript. 
2) Results of attempting the procedure to fit linear pre-and post-transition baselines to the non-sigmoidal demicellization curves.

As described in the main text, we formally applied the procedure demonstrated by Bouchemal et al. on Pluronic F127 to our curves for PS80 and PS20. Under the assumption that also these non-sigmoidal curves contain approximately linear, pre- and post-transition ranges where measured heats are not heats of demicellization, this procedure provides characteristic $\mathrm{cmc}$ ranges (from ST to ET) and enthalpies for a given surfactant product. That means, if these assumptions are met to a good approximation, the results for ST, ET, $\Delta \mathrm{H}$ should not depend on the specific setup of the experiment. Fig. S2 demonstrates that this is not at all the case for PS20 and PS80, highlighting the need for an alternative, more sophisticated approach as presented in the main text.
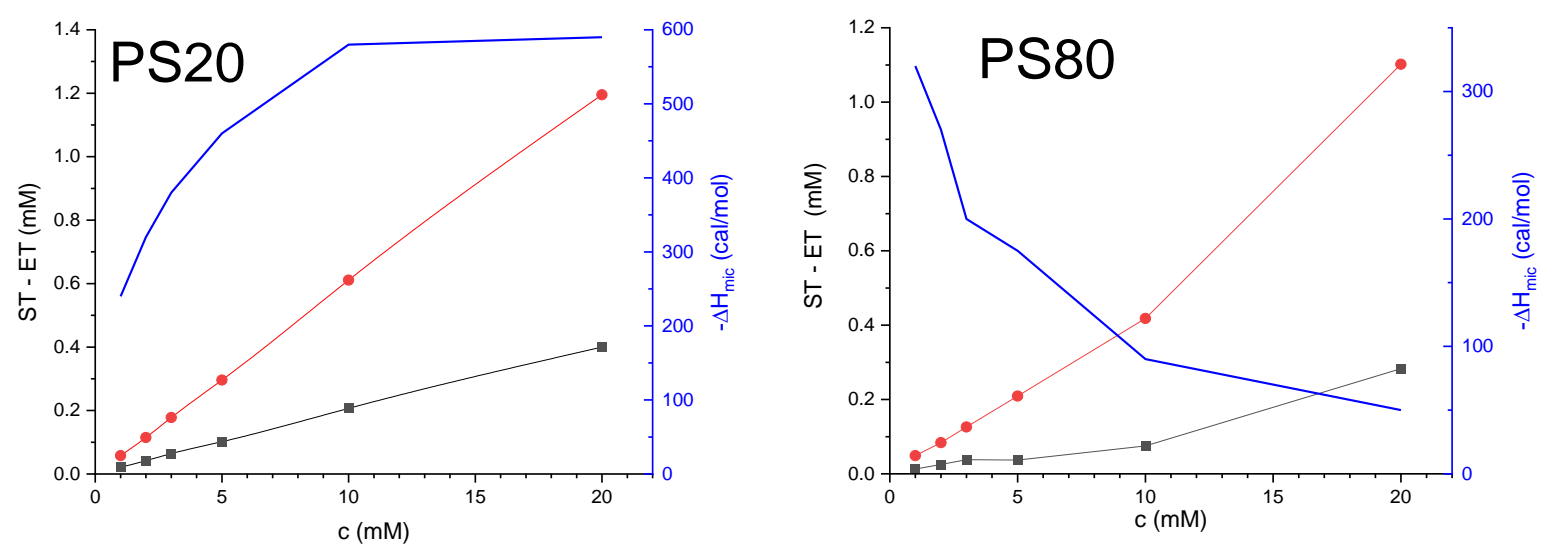

Figure S2. Test of the approach to assign linear pre- and post-transition baselines to nonsigmoidal demicellization curves according to Bouchemal et al. (2009, J. Colloid and Interface Sci. 338:169). Apparent values for the starting (ST, grey squares) and end (ET, red spheres) point of the micellar transition range (left ordinates) as well the enthalpy of micellization, $-\Delta H$ (right ordinates) are plotted as a function of the titrant concentration for series of ITC experiments with PS20 (left panel) and PS80 (right panel). 
3) Analytical solution of equation (9).

We try to solve differential equation (9) for $\mathrm{Q}_{\mathrm{m}}(\mathrm{c})$ :

$$
-N D H(c)=Q_{m}\left(c_{s y r}\right)-Q_{m}(c)-c * Q_{m}^{\prime}(c)\left(1-\frac{c}{c_{s y r}}\right)
$$

For simplification, we write

$$
f(c)=N D H(c)-Q_{m}\left(c_{s y r}\right)
$$

and obtain

$$
Q_{m}(c)=\left(\frac{c^{2}}{c_{s y r}}-c\right) Q_{m}^{\prime}(c)-f(c)
$$

As a boundary condition for the lowest curve, we assume $\mathrm{Q}_{\mathrm{m}}\left(\mathrm{c}_{0}\right)=0$. The solution for the above differential equation is

$$
Q_{m}(c)=\frac{c-c_{s y r}}{c} \int_{c_{0}}^{c} \frac{c_{s y r}}{\left(c_{s y r}-x\right)^{2}} f(x) d x
$$

We can now again substitute $\mathrm{f}(\mathrm{x})$ :

$$
\begin{aligned}
& Q_{m}(c)=\frac{c-c_{s y r}}{c} \int_{c_{0}}^{c} \frac{c_{s y r}}{\left(c_{s y r}-x\right)^{2}}\left(N D H(x)-Q_{m}\left(c_{s y r}\right)\right) d x \\
& Q_{m}(c)=\frac{c-c_{s y r}}{c} \int_{c_{0}}^{c} \frac{c_{s y r}}{\left(c_{s y r}-x\right)^{2}} N D H(x) d x-\frac{c-c_{s y r}}{c} Q_{m}\left(c_{s y r}\right) \int_{c_{0}}^{c} \frac{c_{s y r}}{\left(c_{s y r}-x\right)^{2}} d x \\
& Q_{m}(c)=\frac{c-c_{s y r}}{c} \int_{c_{0}}^{c} \frac{c_{s y r}}{\left(c_{s y r}-x\right)^{2}} N D H(x) d x-\frac{c-c_{s y r}}{c} Q_{m}\left(c_{s y r}\right)\left(\frac{c_{s y r}}{c_{s y r}-c}-\frac{c_{s y r}}{c_{s y r}-c_{0}}\right) \\
& Q_{m}(c)=\frac{c-c_{s y r}}{c} \int_{c_{0}}^{c} \frac{c_{s y r}}{\left(c_{s y r}-x\right)^{2}} N D H(x) d x-\frac{1}{c} \frac{c_{s y r} c_{0}}{c_{s y r}-c_{0}} Q_{m}\left(c_{s y r}\right)+\frac{c_{s y r}}{c_{s y r}-c_{0}} Q_{m}\left(c_{s y r}\right)
\end{aligned}
$$

If $\mathrm{c}_{0} \rightarrow 0$, then

$Q_{m}(c)=\frac{c-c_{s y r}}{c} \int_{0}^{c} \frac{c_{s y r}}{\left(c_{s y r}-x\right)^{2}} \operatorname{NDH}(x) d x+Q_{m}\left(c_{s y r}\right)$ 
In the more general case, where only a part of the $\mathrm{Q}_{\mathrm{m}}\left(\mathrm{c}_{\mathrm{syr}}\right)$ curve is known, we assume $\mathrm{c}_{0} \neq 0$ and $\mathrm{Q}_{\mathrm{m}}\left(\mathrm{c}_{0}\right) \neq 0$. Then the integration above yields:

$$
Q_{m}(c)=\frac{c-c_{s y r}}{c} \int_{c_{0}}^{c} \frac{c_{s y r}}{\left(c_{s y r}-x\right)^{2}} N D H(x) d x-\frac{1}{c} \frac{c_{s y r} c_{0}}{c_{s y r}-c_{0}}\left(Q_{m}\left(c_{s y r}\right)-Q_{m}\left(c_{0}\right)\right)+\frac{c_{s y r} Q_{m}\left(c_{s y r}\right)-c_{0} Q_{m}\left(c_{0}\right)}{c_{s y r}-c_{0}}
$$

We can match the other curves to the first curve and obtain for each curve a pair of the terms

$$
k_{0}=\frac{c_{s y r} c_{0}}{c_{s y r}-c_{0}}\left(Q_{m}\left(c_{s y r}\right)-Q_{m}\left(c_{0}\right)\right) \text { and } \quad k_{1}=\frac{c_{s y r} Q_{m}\left(c_{s y r}\right)-c_{0} Q_{m}\left(c_{0}\right)}{c_{s y r}-c_{0}}
$$

Since $\mathrm{Q}_{\mathrm{m}}\left(\mathrm{c}_{0}\right), \mathrm{c}_{0}$, and $\mathrm{c}_{\mathrm{syr}}$ are know, we can calculate $\mathrm{Q}_{\mathrm{m}}\left(\mathrm{c}_{\mathrm{syr}}\right)$ as

$$
Q_{m}\left(c_{s y r}\right)=\frac{k_{0}\left(c_{s y r}-c_{0}\right)}{c_{s y r} c_{0}}+Q_{m}\left(c_{0}\right) \text { or } \quad Q_{m}\left(c_{s y r}\right)=\frac{k_{1}\left(c_{s y r}-c_{0}\right)+c_{0} Q_{m}\left(c_{0}\right)}{c_{s y r}}
$$

4) Average of total concentration in the cell.

The mole number of surfactant injected from the syringe during a given injection is $\Delta \mathrm{n}^{\text {syr. }}$ :

$$
\Delta n^{s y r}=\Delta V \cdot c^{s y r}
$$

During the injection, the same volume is flowing over from the totally filled cell into the access tube, from where it is assumed to show no significant re-mixing with the cell content. Since the injection is slow compared to the mixing of the injectant with the cell content, it can be approximated that at any time during the injection, the concentration of the overflowing solution agrees with the current average concentration in the cell (including the currently injected material). Hence, the mole number flowing over, $\Delta \mathrm{n}^{\text {over: }}$

$$
\Delta n^{\text {over }}=\Delta V \cdot c
$$

Mass balance requires for the change in content, $\Delta \mathrm{n}^{\text {cell }}$, in the cell of fixed volume, $\mathrm{V}_{0}$, that (switching to a differential form): 


$$
\begin{aligned}
& d n^{\text {cell }}=d n_{s y r}-d n^{\text {over }} \\
& V_{0} d c=c^{s y r} d V-c d V \\
& \frac{d c}{d V}=\frac{c^{s y r}}{V_{0}}-\frac{1}{V_{0}} c
\end{aligned}
$$

A solution of the differential eq. is:

$$
c\left(V_{i n j}\right)=c^{s y r}\left[1-e^{-V_{i n j} / V_{0}}\right]
$$

\section{5) Derivation of equation (12).}

We consider the component $\mathbf{i}$ of a surfactant mixture in equilibrium between an aqueous solution of concentration $\mathrm{c}_{\mathrm{i}}^{\mathrm{aq}}$ and a localization in mixed micelles, where $\mathrm{c}_{\mathrm{i}}^{\mathrm{m}}$ denotes the mole number of micelle-incorporated $\mathbf{i}$ with respect to the total volume of the dispersion. Approximating solution and mixed micelles as pseudo-phases, we obtain from the equilibrium condition that the chemical potential of $\mathbf{i}$ is equal in all coexisting phases:

$$
0=\Delta \mu_{i}^{0}+R T \ln \frac{c_{i}^{m} \cdot 55 M}{c^{m} \cdot c_{i}^{a q}}
$$

where the standard chemical potential change, $\Delta \mu_{\mathrm{i}}{ }^{\circ}$, represents the change between micellar and aqueous states (i.e., it can be assumed to be a constant). The second term covers the effect of the entropy of ideal mixing in the two pseudo-phases, where the activity of $\mathbf{i}$ in the micelle is defined as the mole fraction of $\mathbf{i}$ in the micelle, $\mathrm{c}_{\mathrm{i}}^{\mathrm{m}} / \mathrm{c}^{\mathrm{m}}$, with the total concentration of micellar surfactant

$$
c^{m}=\sum_{i} c_{i}^{m}
$$

The activity of $\mathbf{i}$ in solution is defined as the mole fraction there, which is approximately the aqueous concentration, $\mathrm{c}_{\mathrm{i}}^{\mathrm{aq}}$, divided by the total concentration of water plus surfactant in solution, which is about $55 \mathrm{M}$ for dilute solutions ${ }^{26,27}$. 
In an ideal one-component system consisting of component $\mathbf{i}$ only, $\mathrm{c}_{\mathrm{i}}^{\mathrm{m}} / \mathrm{c}^{\mathrm{m}}=1$ and $\mathrm{c}_{\mathrm{i}}^{\mathrm{aq}}=\mathrm{cmc}_{\mathrm{i}}$, so that with eq. (5.1) we find for the standard chemical potential change, which should be conserved in the multi-component system as well:

$$
\Delta \mu_{i}^{0}=-R T \ln \frac{55 M}{c m c_{i}}
$$

and combining eq. (5.1) with eq. (5.3) we obtain:

$$
0=\ln \frac{c m c_{i}}{55 M} \frac{c_{i}^{m} 55 M}{c^{m} c_{i}^{a q}}
$$

and, hence,

$$
\frac{c_{i}^{a q}}{c_{i}^{m}}=\frac{c m c_{i}}{c^{m}}(5.5) .
$$

Defining the total concentration of $\mathbf{i}$ that results in half of $\mathbf{i}$ to be located in mixed micelles as $\mathrm{c}_{50}(\mathrm{i}),(5.5)$ implies that

$$
c_{50}(i) \equiv c_{i}\left(c_{i}^{m}=c_{i}^{a q}\right)=c_{i}\left(c^{m}=c m c_{i}\right)
$$

That means, each component $\mathbf{i}$ is half micelle-bound when the sum of the concentrations of all components, $\mathrm{c}^{\mathrm{m}}$, reaches the $\mathrm{cmc}$ of this individual component $\mathbf{i}$. In other words, considering the system with increasing total surfactant concentration $\mathrm{c}^{\mathrm{m}}$, the components enter the mixed micelles one by one with increasing $\mathrm{cmc}_{\mathrm{i}}$. 